\title{
A Study of Solutions for Thick Plate Bending Problems Based on Boundary Integral Method
}

\author{
Yingjie Chen ${ }^{1}$, Chao Wang ${ }^{2}$, Jianxun Yang ${ }^{1}$, Yingwei Song ${ }^{1}$ and Teng Teng ${ }^{1}$ \\ ${ }^{1}$ Yanshan University, Department of Civil Engineering, 066004 Qin Huangdao, China \\ ${ }^{2}$ Shen Kan Qinhuangdao Engineering \& Technology Corporation, MCC, The First Engineering \\ Company, 066001 Qinhuangdao, China
}

\begin{abstract}
Keywords: thick plate theory; the boundary integral method; forced vibration; the boundary conditions; governing equation; deflection surface equation

Abstract: Based on Reissner theory, this paper investigates the vibration problems of rectangular thick paltes by applying boundary integral method. This paper presents the concept of the quasi basic system, applies the boundary integral method in it and the actual system, and derives the governing equation and the flexible equation of the forced vibration of rectangular thick plates under a concentrated load with three kinds of boundary conditions respectively. Matlab and Ansys structural analysis software are used for analyzing the equation with numerical calculation and simulation analysis. The result of this paper is used to prove the correctness of the new method, which has solved forced vibration problem of rectangular thick plate.
\end{abstract}

\section{Introduction}

In modern industrial construction, the theory of computation for plate is very important, but the classical plate theory has relatively great errors when solving problems of thick plate. In recent years, some scholars found that applying classical plate theory and thick plate theory separately in solving problems of plate will make errors when the ratio of thickness for spans varies in a certain range (Long, 2012), so new theories and methods for solving the problems of plate are urgent.

Common methods for solving thick plate bending problems have larger errors in practical projects. There are many ways for solving thick plate bending problems which have flowed more than one century, but shcolars still aim to explore the exact solution. Scholars at home also have lots of studies about thick plate problems (Hosseini, 2005; Khorshidi, 2009; Gorman, 1997; Ohya, 2006).

This article, based on Reissner theory, turns the vibration problem of thick plate into static problem with Dalembert's principle which can avoid the simplifying solution of high-order differential equation effectively, and then obtains exact solutions of thick plate vibration problem.

\section{The basic theory of bending rectangular plate}

The following sections will introduce Reissner plate theory and the boundary conditions of bending rectangular thick plates, which take into account the effects of transverse shear deformation and compression deformation on rectangular thick plate form.

\subsection{The Reissner theory of bending thick plate}

As shown in Fig1, a rectangular thick plate acted has transverse external load, and stresses on lateral sides, such as $\sigma_{x}, \tau_{x y}, \tau_{x z} ; \sigma_{y}, \tau_{y x}, \tau_{y z}$. According to the Reissner theory:

$$
\begin{aligned}
& \sigma_{x}=\frac{12 M_{x}}{h^{3}} z \\
& \sigma_{y}=\frac{12 M_{y}}{h^{3}} z \\
& \tau_{x y}=\frac{12 M_{x y}}{h^{3}} z
\end{aligned}
$$




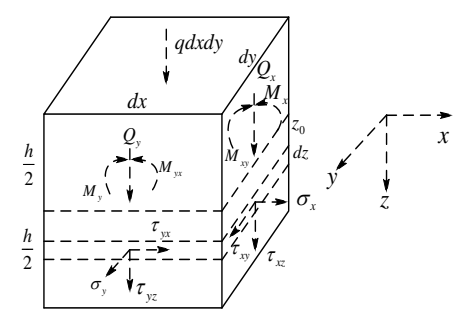

Fig1. the stresses distribution of plate unit

When substituting (1) (3) in the differential equilibrium equation, the shear forces of upper and lower surfaces are $0, \tau_{x z}$, and $\tau_{y z}$ are obtained. With the equilibrium equation, $\sigma_{z}$ can be obtained with the boundary conditions of upper and lower surfaces. The force equilibrium of rectangular thick plate needs to be considered. Surface of the unit is affected by the working of distributed load q. The shear force, bending moment and torque are distributed in each lateral surface.

Obviously, all forces of $\mathrm{z}$ axis which act on the rectangular thick plate unit are 0 . The sum of torques of $\mathrm{x}$ axis and $\mathrm{y}$ axis which are generated by distributed load and shearing force on each lateral surfaces, is 0 . According to the relations mentioned, the differential equilibrium equation of bending rectangular thick plate can be derived from Reissner theory, which is obvious different from the classical plate theory.

For convenient calculating, the equation can be rewitten as (4), which is affected by the working together of distributed load (q), concentrated load (P) and concentrated bending moment $\left(M_{x 1}\right)$.

$$
\begin{aligned}
D \nabla^{4} \omega & =q-\frac{h^{2}}{10} \frac{2-v}{1-v} \nabla^{2} q+P \delta\left(x-x_{0}, y-y_{0}\right) \\
& -\frac{P h^{2}}{10} \frac{2-v}{1-v} \nabla^{2} \delta\left(x-x_{0}, y-y_{0}\right) \\
& +M_{x 1} \delta_{x}^{\prime}\left(x-x_{1}, y-y_{1}\right)-\frac{M_{x 1} h^{2}}{10} \frac{2-v}{1-v} \nabla^{2} \delta_{x}^{\prime}\left(x-x_{1}, y-y_{1}\right)
\end{aligned}
$$

The expressions of shear force, bending moment, torque and torsional angle of curving thick plate, which also means the governing equation of forced vibration of curving rectangular thick plate, can be deduced according to Dalembert's principle and formula (4), with the acting of simple harmonic load (no resistance).

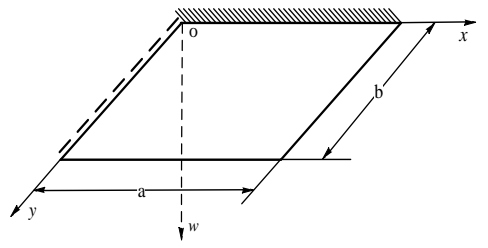

Fig2. the rectangular plate with different boundary conditions

Torques and shear forces of free boundary of thick plate are not necessary to be replaced by equivalent shear force, which is different from sheet.

As shown in Fig2, each side's boundary conditions of rectangular thick plate are:

Simple supported edge $x=0: w=\omega_{y}=M_{x}=0$

Free edge $x=a: \quad Q_{x}=M_{x y}=M_{x}=0$

Fixed edge $y=0: \quad w=\omega_{y}=\omega_{x}=0$

\subsection{Boundary integral method}

Many studies, based on the classical propositional theorem, have been conducted by scholars. Before 1980, the reciprocal theorem has been limited in the same elastomer with same boundary conditions. Afterwards, Professor Fu Baolian has made certain achievements in theoretical research and application of reciprocal theorem. His research have been agreed by renowned scholars at home and abroad. After a series of researches, he finally obtained systematic structural analysis method for the study of thick plate, named boundary integral method. The method has expanded the application area of reciprocal theorem, and developed its new applications.

The boundary integral method of thick plate's forced vibration can be simply described as 
follows. Applying reciprocal theorem in amplitude hypothetical basic system and corresponding amplitude actual system, the amplitude flexible equation of actual system can be obtained. Then calculating the executive equation of flexible equation after getting it, the corresponding parameter can be substituted in the equation and the the solution can be obtained.

\subsection{Parametrix of rectangular thick plate in boundary integral method}

Rectangular thick plate which simply supported by four simple edges, which is only influenced by transverse two-dimensional DE carat function, is treated as parametrix system. As shown in Fig 3, its solution is named as parametrix solution. In the process of derivation, rectangular thick plate with four simply supported egdes, under the working of one unit's concentrated load, can be chose as the parametrix system.

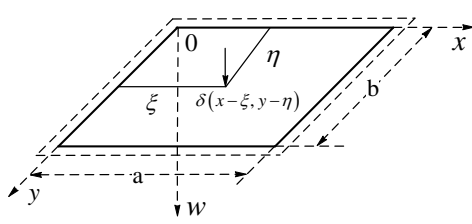

Fig3. parametrix system of bending rectangular thick plate

Flexible governing equation of static parametrix solution of rectangular thick plate:

$$
D \nabla^{4} w_{1}=\delta(x-\xi, y-\eta)
$$

According to above, the right side of equation (5) should consider the effects of plate thickness when there are only effects of concentrated load on it. After calculating, it satisfies the differential equation without mechanical meaning when only the effect of transverse two-dimensional DE carat function is considered.

Function $\delta(x-\xi, y-\eta)$, which acts on $\operatorname{point}(\xi, \eta)$ of the plate, is called parametrix load.

\section{Forced vibration of bending rectangular plate in different boundary conditions under the working of concentrated load}

Forced vibration equation of bending rectangular thick plate with different boundary conditions will be discussed next, under the working of concentrated load which is composed of edges which are simply supported, fixed and free. The flexible equation of corresponding boundary conditions and executive equation of boundary condition will be deduced by applying the boundary integral method, and Matlab numerical calculation and Ansys finite element simulation will be used for comparison and test.

\subsection{Rectangular thick plate with four simply supported edges}

\subsubsection{Flexible equation}

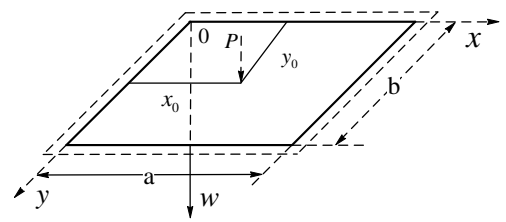

Fig4. The amplitude actual system of bending rectangular thick plate with four simply supported edges under the working of concentrated harmonic load

The amplitude actual system of bending rectangular thick plate simply supported by four edges under the working of concentrated harmonic load is shown as Fig4. Applying the boundary integral method in hypothetical basic system as shown in Fig3 and amplitude actual system with four simply supported edges as shown in Fig4, amplitude flexible equation about forced vibration of bending rectangular thick plate with four simply suppored edges under the effects of concentrated load can be deduced. As shown below: 


$$
\begin{aligned}
w\left(\xi, \eta, a-x_{0}, y_{0}\right)= & -\frac{2 P}{D b} \sum_{n=1,2}^{\infty} \frac{1}{k_{n}^{2}-\lambda_{n}^{2}}\left[\frac{\sinh k_{n}\left(a-x_{0}\right)}{k_{n} \sinh k_{n} a} \sinh k_{n} \xi\right. \\
& \left.-\frac{\sinh \lambda_{n}\left(a-x_{0}\right)^{2}}{\lambda_{n} \sinh \lambda_{n} a} \sinh \lambda \lambda_{n} \xi\right] \cdot \sin \beta_{n} y_{0} \sin \beta_{n} \eta \\
& +\frac{2 P}{D b} \frac{k h^{2}}{10} \sum_{n=1,1}^{\infty} \frac{1}{k_{n}^{2}-\lambda^{2}}\left[\left(k_{n}^{2}-\beta_{n}^{2}\right) \frac{\sinh k_{n}\left(a-x_{0}\right)}{k_{n} \sinh k_{n} a} \sinh k_{n} \xi\right. \\
& \left.-\left(\lambda_{n}^{2}-\beta_{n}^{2}\right) \frac{\sinh \lambda_{n}\left(a-x_{0}\right)}{\lambda_{n} \sinh \lambda_{n} a} \sinh \lambda_{n} \xi\right] \cdot \sin \beta_{n} y_{0} \sin \beta_{n} \eta
\end{aligned}
$$

\subsubsection{Stress function}

First, stress function should be assumed and verified.

And $E_{n}, F_{n}, G_{m}, H_{m}$ can be confirmed, because the bending moment of bending rectangular thick plate simply supported by four edges is equal to 0 . Then flexible equation and hypothetical stress function are substituted in the expression of the plate bending moment amplitude, and $E_{0}, F_{0}, G_{0}, H_{0}$ are defined because the twist angle of bending rectangular thick plate simply supported by four edges is equal to 0 .

$$
\begin{aligned}
\varphi(\xi, \eta) & =\sum_{n=0,1,2}^{\infty}\left[E_{n} \cosh \delta_{n} \xi+F_{n} \cosh \delta_{n}(a-\xi)\right] \cos \beta_{n} \eta \\
& +\sum_{m=0,1,2}^{\infty}\left[G_{n} \cosh \gamma_{m} \eta+H_{n} \cosh \gamma_{m}(b-\eta)\right] \cos \alpha_{m} \xi
\end{aligned}
$$

In the formula:

$$
\delta_{n}=\sqrt{\beta_{n}^{2}+\frac{10}{h^{2}}}, \gamma_{m}=\sqrt{\alpha_{m}^{2}+\frac{10}{h^{2}}}
$$

\subsubsection{Numerical calculation and finite element analysis}

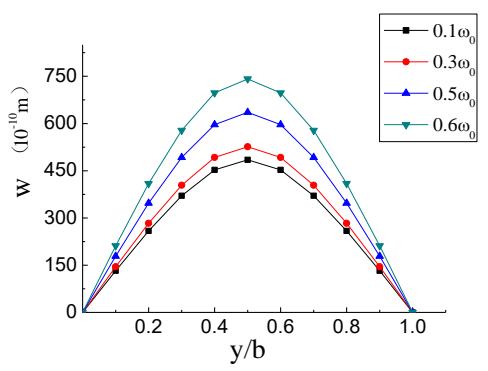

Fig5. simply supported by four edges, $h / a=0.1, x / a=0.3$, value of line of deflection The basic parameters of the system have been determined (the calculation method of fundamental frequency of rectangular thick plate can be ensured through consulting Mechanical Vibration Handbook (Tang,2000)). Then it can be solved after assigning value directly to the flexible equation about forced vibration of rectangular thick plate with four simply supported edges on Matlab platform. Ansys, the finite element analysis software, is used for simulating with the top 50 items for the formula. The results of numerical calculation and finite element simulation are shown in Fig5.

\subsection{Bending rectangular thick plate with fixed opposite edges and simply supported opposite edges}

\subsubsection{Flexible equation}

Bending rectangular thick plate with fixed opposite edges and simply supported opposite edges under the working of concentrated harmonic load are shown as in Fig6. Replacing the bending moment constraints of two fixed edges with distributed bending moments $M_{x 0}$ and $M_{x a}$, the equivalent diagram of amplitude actual system can be obtained as shown in Fig7. Suppose:

$$
M_{x 0}=\sum_{n=1,2}^{\infty} A_{n} \sin \beta_{n} y
$$




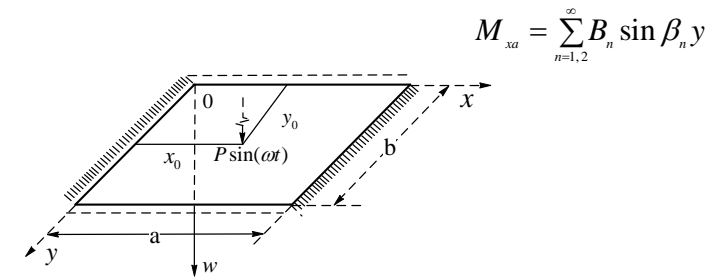

Fig6. Amplitude actual system of bending rectangular thick plate with fixed opposite edges and simply supported opposite edges

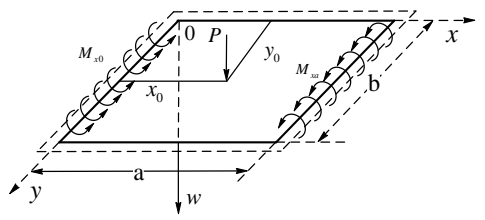

Fig7. Equivalent diagram of amplitude actual system of bending rectangular thick plate with fixed opposite edges and simply supported opposite edges

Flexible equation can be obtained by applying boundary integral method in equivalent diagram of amplitude actual system as shown in Fig7 and amplitude hypothetical basic system as shown in Fig3.

\subsubsection{Stress function}

The bending rectangular thick plate with fixed opposite edges and simply supported opposite edges can be regard as bending rectangular thick plate with four simply suppored edges which is affected by distributional bending moment on the opposite edges. The stress function:

$$
\varphi(\xi, \eta)=\sum_{n=1,2}^{\infty}\left[B_{n} \cosh \delta_{n} \xi-A_{n} \cosh \delta_{n}(a-\xi)\right] \frac{\beta_{n}}{\delta_{n} \sinh \delta_{n} a} \beta_{n} \eta
$$

\subsubsection{Boundary condition}

According to boundary condition, the following formula should be:

$$
\omega_{\xi \xi 0}=\omega_{\xi \xi a}=0
$$

The executive equation of boundary condition can be deduced by calculating with flexible equation and stress function (11).

\subsubsection{Numerical calculation and finite element analysis}

Although basic parameters are the same with the previous section, this section has something different in calculation. Several corresponding parameters need be obtained through governing equation firstly, then the flexible equation. In order to ensure the convergence, this paper applies basic concept of solving equations in $A_{n}$ and $B_{n}$, which include 50 values respectively. After solving the two groups of values and substituting them in the flexible equation, arithmetic solutions are obtained as shown in Tab1.

Tab1. fixed opposite edges and simply supported opposite edges, $h / a=0.1, x / a=0.5$, value of line of deflection

\begin{tabular}{|c|c|c|c|c|c|c|}
\hline \multirow{2}{*}{$y / b$} & \multicolumn{2}{|c|}{$0.1 \omega_{0}$} & \multicolumn{2}{c|}{$0.3 \omega_{0}$} & \multicolumn{2}{c|}{$0.5 \omega_{0}$} \\
\cline { 2 - 7 } & Ansys & $\begin{array}{c}\text { Our } \\
\text { method }\end{array}$ & Ansys & $\begin{array}{c}\text { Our } \\
\text { method }\end{array}$ & Ansys & $\begin{array}{c}\text { Our } \\
\text { method }\end{array}$ \\
\hline 0 & 0 & 0 & 0 & 0 & 0 & 0 \\
\hline 0.1 & 92.68 & 94.08 & 102.54 & 103.89 & 127.86 & 129.90 \\
\hline 0.2 & 188.16 & 190.83 & 206.62 & 209.60 & 255.45 & 259.32 \\
\hline 0.3 & 286.71 & 290.28 & 312.34 & 316.34 & 380.02 & 385.22 \\
\hline 0.4 & 383.50 & 387.76 & 413.88 & 418.62 & 493.94 & 500.08 \\
\hline 0.5 & 491.27 & 504.80 & 523.16 & 537.36 & 607.11 & 623.24 \\
\hline
\end{tabular}




\subsection{Rectangular thick plate with four fixed edges}

\subsubsection{Flexible equation}

Bending rectangular thick plate with four fixed edges affected by concentrated harmonic load as shown in Fig8. Removing bending moment constraints of four fixed edges, and replacing with distributed bending moments $M_{x 0}, M_{x a}, M_{y 0}$ and $M_{y b}$, equivalent diagram of amplitude actual system of rectangular thick plate is obtained as shown in Fig9. Suppose:

$$
\begin{aligned}
& M_{x 0}=\sum_{n=1,2}^{\infty} A_{n} \sin \beta_{n} y \\
& M_{x a}=\sum_{n=1,2}^{\infty} B_{n} \sin \beta_{n} y \\
& M_{y 0}=\sum_{m=1,2}^{\infty} C_{m} \sin \alpha_{m} x \\
& M_{y b}=\sum_{m=1,2}^{\infty} D_{m} \sin \alpha_{m} x
\end{aligned}
$$

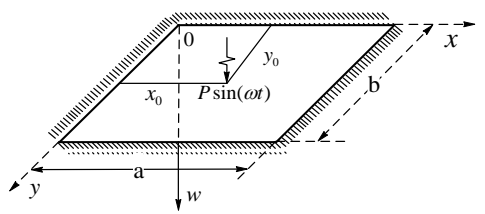

Fig8. Actual system of bending rectangular plate with four edges

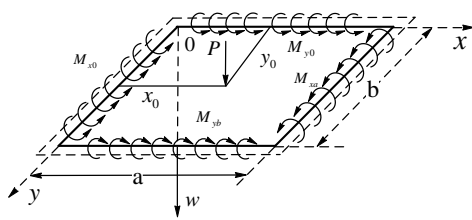

Fig9. Equivalent diagram of amplitude actual system of bending rectangular thick plate with four edges

Flexible equation can be obtained by applying boundary integral method in equivalent diagram of amplitude actual system as shown in Fig9 and amplitude hypothetical basic system as shown in Fig3.

\subsubsection{Stress function}

The process of solving stress function in the boundary condition is basically same to 3.2 .2 , so

$$
\begin{aligned}
\varphi(\xi, \eta) & =\sum_{n=1,2}^{\infty}\left[B_{n} \cosh \delta_{n} \xi-A_{n} \cosh \delta_{n}(a-\xi)\right] \frac{\beta_{n}}{\delta_{n} \sinh \delta_{n} a} \cos \beta_{n} \eta \\
& -\sum_{m=1,2}^{\infty}\left[D_{m} \cosh \gamma_{m} \eta-A_{m} \cosh \gamma_{m}(b-\eta)\right] \frac{\alpha_{m}}{\gamma_{m} \sinh \gamma_{m} b} \cos \alpha_{m} \xi
\end{aligned}
$$

\subsubsection{Boundary condition}

According to boundary condition, following formula should be meet:

$$
\omega_{\xi \xi 0}=\omega_{\xi \xi a}=\omega_{\eta \eta 0}=\omega_{\eta \eta b}=0
$$

The executive equation of boundary condition can be deduced by calculating with flexible equation and stress function (17).

\subsubsection{Numerical calculation and finite element analysis}

The basic parameters and calculation method of this section are basically same to 3.2.4.

\section{Conclusion}

The paper applies boundary integral method in the actual system and amplitude hypothetical basic 
system of rectangular thick plate, solves the governing equation and flexible equation in different boundary conditions, and verifies the correctness of the equation through numerical calculation and finite element analysis. The solution, for the problem of rectangular thick plates' forced vibration, simplifies the solving process. The method forms a set of independent problem-solving thoughts, which is easier to understand and is simple and efficient in the process of application.

\section{References}

[1] Shuyao Long, Chen Jiang. Journal of Hunan University (Natural Science), 39, 37-41 (2012)

[2] Hosseini Hashemi S, Arsanjani M.. International Jounrnal of Solid Sand Structures, 42, 819-853 (2005)

[3] Hosseini Hashemi S, Khorshidi K.. JSV, 322, 883-900 (2009)

[4] Gorman DJ.. JSV, 207, 335-350 (1997)

[5] Ohya F,Ueda M,et al.,JSV, 289, 1-24 (2006)

[6] Hengling Tang, Weide Qu, Mechanical vibration handbook (China Machine Press, Beijing, 2000)

[7] $\mathrm{Fu}$ Baolian. The reciprocal theorem of bending of rectangular thick plates and its applications, (National Defense Industry Press, Beijing, 2014) 